\title{
Using Gravitational Lenses to Detect Gravitational Waves ${ }^{\dagger}$
}

\author{
B. Allen ${ }^{1}$
}

Gravitational lenses could be used to detect gravitational waves, because a gravitational wave affects the travel-time of a light ray. In a gravitational lens, this effect produces time-delays between the different images. Thus the bending of light, which was the first experimental confirmation of Einstein's theory, can be used to search for gravitational waves, which are the most poorly confirmed aspect of that same theory. Applying this method to the gravitational lens $0957+561$ gives new upper bounds on the amplitude of low-frequency gravitational waves in the universe, and new limits on the energy-density during an early "inflationary" phase.

\section{INTRODUCTION}

The deflection of star-light by the gravitational field of the sun was first observed in 1919; it was the first successful prediction of Einstein's general theory [1]. This bending of light is now routinely observed in gravitational lenses. In a gravitational lens, light from a distant quasar is bent as it travels around a galaxy or cluster of galaxies (see Figure 1). The result of this bending is that an observer on the earth, looking in the sky, sees two (or more) images of the same quasar (see Figure 2). Since the discovery of the first of these "double quasars" $(0957+561)$ in 1979 , about thirty such gravitational lenses have been found [2].

This Essay received the First Award from the Gravity Research Foundation, 1990 Ed.

1 Department of Physics, University of Wisconsin at Milwaukee, P.O. Box 413, Milwaukee, Wisconsin 53201, USA 


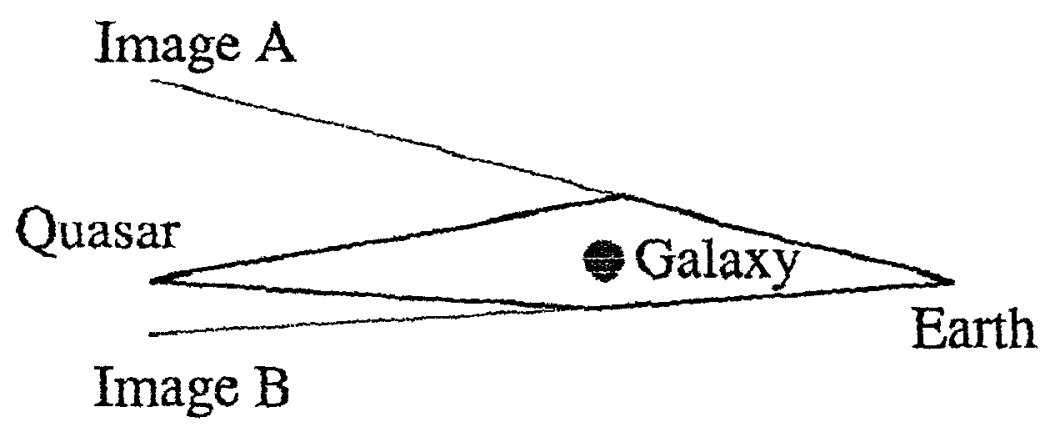

Figure 1. Schematic diagram of gravitational lens, showing paths of light-rays. (a) The lens $0957+561$ is a large galaxy at a redshift of 0.36 , imaging a quasar at a redshift at 1.4 .

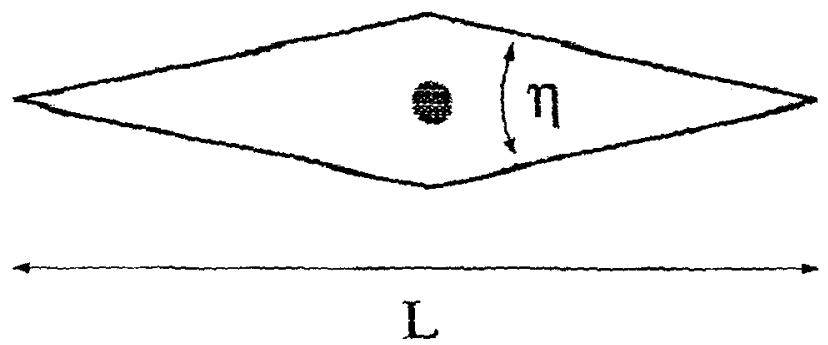

(b) Idealized model used for gravitational-wave calculation has paths composed of four symunetrically-placed segments.

A second, more poorly verified prediction of Einstein's general theory is that weak gravitational disturbances, propagating at the speed of light, carry energy and momentum away from an accelerated body. Only a single indirect observation of these waves has been made, in the binary pulsar PSR1913+16. This rapidly orbiting system is slowly sppeding up, and the corresponding loss of energy is taking place at exactly the rate predicted by General Relativity. The usual interpretation is that the binary pulsar is losing energy in the form of gravitational radiation. An intensive effort is now underway to construct a new generation of sensitive gravitational wave detectors, to permit direct observation of this type of radiation [3].

The main point of this essay is that gravitational lenses can serve as gravitational wave detectors. These "detectors" are 22 orders of magnitude 


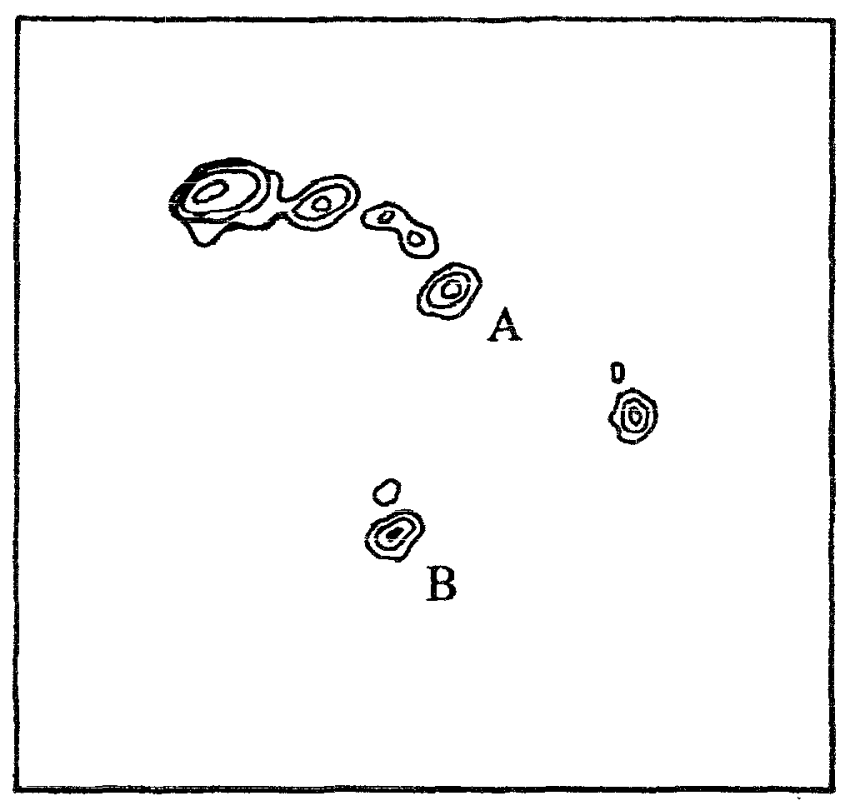

Figure 2. Radio image of $0957+561$, courtesy of the NRAO/AUI, observed by P. E. Greenfield, D. H. Roberts, and B. F. Burke. Field of view is 19.2 arcseconds square. The north (A) and south (B) point-like objects are images of the quasar, and the weak image just north of $B$ is the lensing galaxy.

larger than any of the existing or contemplated instruments, and consequently they are sensitive to much lower frequencies. However, in many respects they are similar to interferometric gravitational-wave detectors [3].

\section{GRAVITATIONAL LENSES AS GRAVITATIONAL WAVE DETEC- TORS}

The use of gravitational lenses as gravitational wave detectors is possible because gravitational waves would produce a time-delay between the different images in a gravitational lens. This time-delay $\Delta t$ can be measured, because the source quasar fluctuates in brightness, producing images whose brightness variations are correlated, with a time-shift [4].

Figure 3 shows the intensities $s_{A}(t)$ and $s_{B}(t)$ of the two images in $0957+561$ over an eight-year period. The time-delay can be determined from the correlation function $\sigma(\Delta t)=\int s_{A}(t+\Delta t) s_{B}(t) d t$. The function 


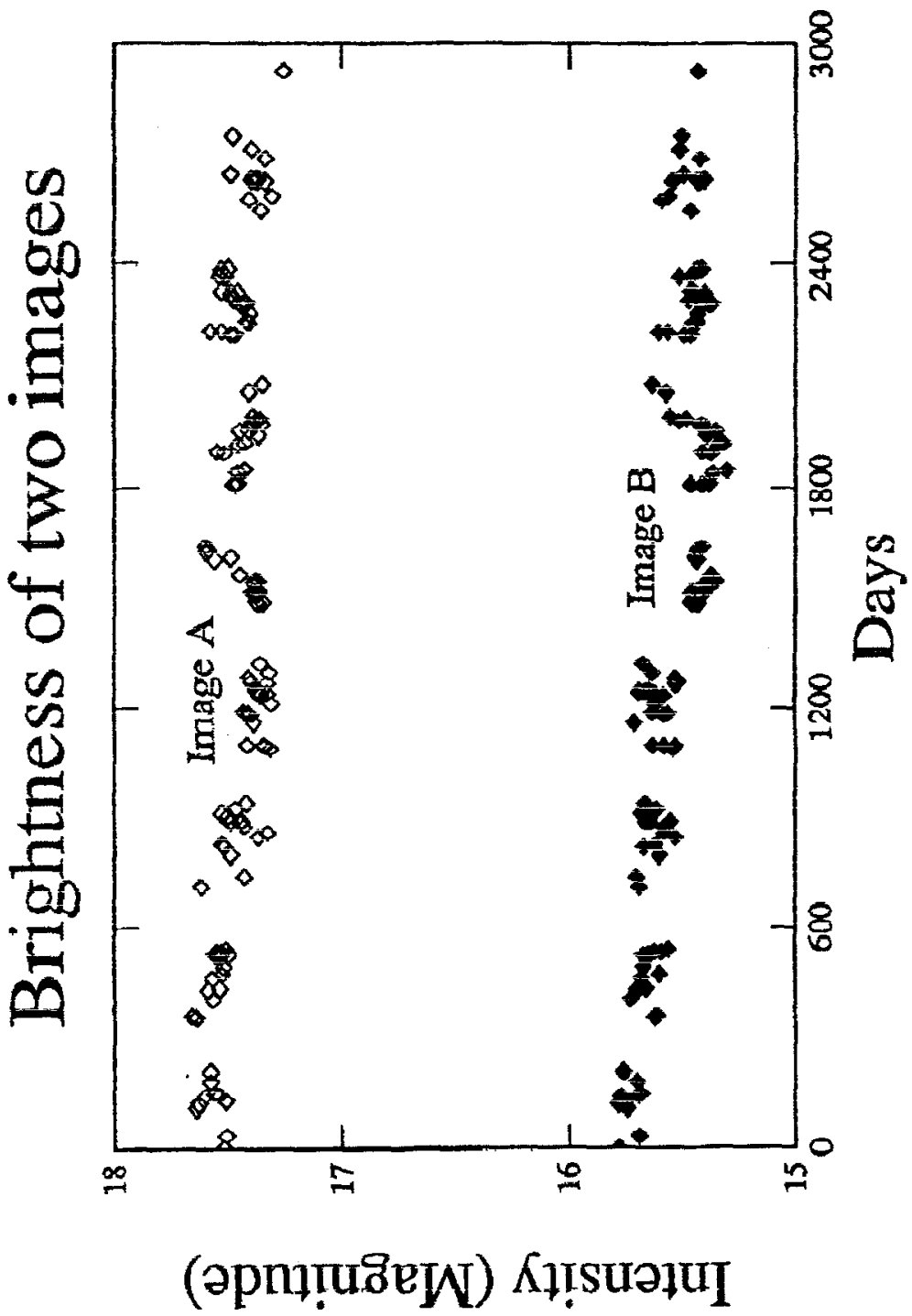

Figure 3. Optical intensities (magnitudes) of A and B images in $0957+$ 561 , from reference [4]. To separate the curves, the magnitude of image $B$ has been reduced by 1 . It is easy to see from this data that image $B$ is delayed by 420 days from image $A$. The origin on the $\mathrm{x}$-axis is 16 November 1979. 
$\sigma(\Delta t)$ is sharply peaked at $\Delta t=420$ days [4], clearly establishing that the two images show the same source.

The 420-day delay is about what one would expect due to path length differences,

$$
\Delta t \approx L(1-\cos \eta) \approx L \eta^{2} \approx 1 \text { year }
$$

since the distance to the quasar is about $L=10^{10}$ light-years, and the angle between the two images is $\eta=3 \cdot 10^{-5}$ radians. (Throughout this essay we use units in which the speed of light $c=1$.)

A gravitational wave affects the time-delay because it perturbs the metric tensor and hence modifies the path length of the two light rays. The metric is given by

$$
g_{a b}=\eta_{a b}+h_{a b}
$$

where $\eta_{a b}$ is the Minkowski metric, and $h_{a b}$ is a small perturbation. In transverse-traceless synchronous gauge, a plane gravitational wave of angular frequency $\omega$, traveling in the $\hat{z}$ direction, produces a metric perturbation

$$
h_{a b}=\left(\begin{array}{cccc}
0 & 0 & 0 & 0 \\
0 & h_{+} & h_{\times} & 0 \\
0 & h_{\times} & -h_{+} & 0 \\
0 & 0 & 0 & 0
\end{array}\right) \cos (\omega(t-z)) .
$$

In general, a wave moving in direction $\hat{k}$ is characterized by the amplitudes of its two polarizations, denoted here by $h_{\times}$and $h_{+}$. We use

$$
h^{2}=h_{a b} h^{a b}=2\left(h_{\times}^{2}+h_{+}^{2}\right),
$$

as a measure of overall amplitude.

One can calculate time-delay $\Delta t$ by examining the influence of the metric perturbation on the equation of motion of a null geodesic [5]. For the symmetric model of the gravitational lens shown in Figure 1(b), one obtains [6]

$$
\begin{aligned}
\Delta t= & 2 \eta \omega^{-1} \sin (\omega t) \sin \theta \sin ^{2}\left(\frac{\omega L}{2} \sin ^{2} \frac{\theta}{2}\right) \times \\
& \times\left[h_{+} \cos \phi+h_{\times} \sin \phi \csc ^{2} \frac{\theta}{2}\right],
\end{aligned}
$$

where we have assumed a small angle between images, $\eta \ll 1$. (Compared to the laboratory interferometric detectors, the gravitational lens suffers from poor design. Since the two "arms" are not perpendicular, the sensitivity is reduced by a factor of $\eta$.) The angle between the vector $\hat{k}$ and 
the line-of-sight to the lens is denoted by $\theta$, and $\phi$ denotes the azimuthal angle of $\hat{k}$ about the line-of-sight, measured from a line passing between the two quasar images.

Now consider the effect of an unpolarized isotropic stochastic background of gravitational waves at frequency $\omega$. (The amplitudes, directions, and phases of the different Fourier components are uniformly distributed random variables, so the distribution is characterized by the mean-square amplitude $\left\langle h^{2}\right\rangle$.) Because the time delay $\Delta t$ is linear in $h_{+}$and $h_{\times}$, its mean value vanishes, but its r.m.s value is non-zero:

$$
\Delta t_{\mathrm{rms}}=\sqrt{\left\langle(\Delta t)^{2}\right\rangle}=\eta L f(\omega L) \sqrt{\left\langle h^{2}\right\rangle} .
$$

The frequency response function $f(\omega L)$ is obtained by averaging equation $(5)$,

$$
f^{2}(x)=x^{-2} \int_{0}^{2} d s \sin ^{4}\left(\frac{x s}{4}\right)(2-s)(s+4 / s),
$$

and is shown in Figure 4. This function is peaked around $\omega L \approx 2 \pi$, which means that the detector's sensitivity is greatest at wavelengths comparable to the overall size of the lens. At higher frequencies, the sensitivity drops off as $f(\omega L) \approx 1 / \omega L$.

The measured time delay in the gravitational lens $0957+561$ can now be used to put an upper limit on the amplitude of gravitational waves in the frequency range $\omega>2 \pi \times 10^{-18} \mathrm{~Hz}$. The amplitude of the gravitational waves must be less than

$$
h<2 \times 10^{-5}\left[\frac{\omega}{2 \times 10^{-17}}\right] \frac{\mathrm{sec}}{\mathrm{radian}},
$$

or the expected time delay $\Delta t_{\text {rms }}$ would be greater than the measured value of 420 days.

At the low end of the frequency range, these limits are more than an order of magnitude better than any existing limits on the gravitationalwave amplitude. Because a period of inflation would have produced a great deal of ultra-low frequency gravitational radiation [7], one can give an improved limit [6] on the energy density during an early phase of inflationary expansion,

$$
\rho_{\text {inflation }}<10^{84} \mathrm{gm} \mathrm{cm}^{-3} \text {. }
$$

In units with $\hbar=c=1$, this energy-density limit is $\rho_{\text {inflation }}<(5$. $\left.10^{16} \mathrm{Gev}\right)^{4}$.

The potential of this method will clearly take some time to exploit. Simple estimates show that approximately 15,000 lenses ought to be visible 


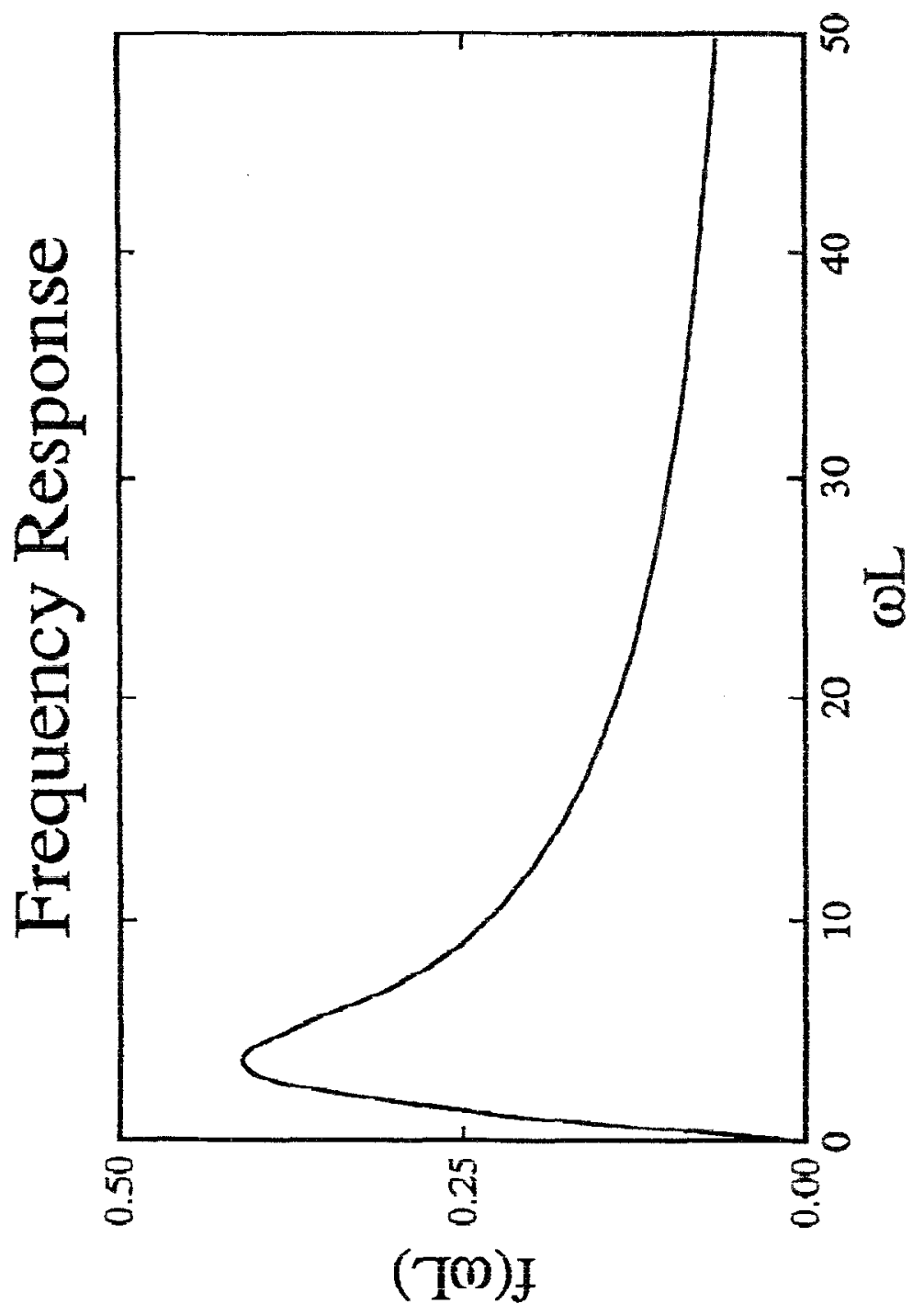

Figure 4. Frequency response of a gravitational lens to unpolarized isotropic gravitational waves [equation (6)]. The response is peaked at angular frequencies $\omega \approx 1 / L$ where $L$ is the distance to the lens. 
with current telescopes [8], and in fact about one new gravitational lens is being discovered every month. When the time delays in some of these systems are measured, they will permit us to place more stringent bounds on the gravitational wave amplitudes in our universe. For this purpose, the most useful systems are those which are extremely symmetric, so that the time-delay due to path length difference is small. One may also make good use of lenses which form multiple images, for in these systems the additional data permits the mass distribution to be tightly constrained. By picking lenses at different distances away from us, one can also modify the wavelength for which the detector has maximum sensitivity.

Another potential use of these very large gravitational wave detectors is to probe the fluctuations in the mass density of the universe (the scalar gravitational perturbations) but lack of space does not permit us to take up this subject here.

Note added:

Since this paper was completed, it has been brought to my attention that the Sachs-Wolfs formula, which was used (in Ref. 6) to calculated the time delay [equation (5)] neglects the spatial perturbation of the geodesics induced by the gravitational wave. In the case where the amplitude of the gravitational wave $h$ is small $(h<\eta)$ the effects of this approximation are negligible, and equation (6) for the r.m.s. time-delay can be applied. However if $h$ is larger than $\eta$, the spatial motion of the geodesics which form the two images becomes significant. While the r.m.s. time-delay is still well-approximated by equation (6), one side effect of the gravitational perturbation is to change the angle $\eta$ between the images, usually by increasing it. The relative intensities of the two images also change.

Thus it becomes important to determine if the time delay due to the gravitational wave can be larger than of order $L \eta^{2}$. If so, then the ideas of this paper have practical applications. If not, it may be difficult, or even impossible, to separate the "intrinsic" geometrical time delay from the delay due to the effects of the gravitational wave. In this latter case, the idea is much harder to apply, for it would involve detailed modeling of each gravitational-lens system.

\section{ACKNOWLEDGEMENT}

This work has been partly supported by NSF grant PHY89-03027.

\section{REFERENCES}

1. Dyson, F. W., Eddington, A. S. E., and Davidson, C. R. (1920). Phil. Trans. Roy. Soc., A220, 291. 
2. Turner, E. (1989). In 14th Texas Symposium on Relativistic Astrophysics, E. J. Fenyves, ed. (New York Academy of Science, New York).

3. Thorne, K. (1989). Gravitational Radiation, (Cambridge University Press, Cambridge).

4. Vanderriest, C., Schneider, J., Herpe, G., Chevreton, M., Moles, M., and Wlérick, G. (1989). Astron. Astrophys., 215, 1.

5. The method of calculation is the same as in Sachs, R., and Wolfe, A. (1967). Ap. $J ., 147,173$.

6. Allen, B. (1989). Phys. Rev. Lett., 63, 2017.

7. Allen, B. (1988). Phys. Rev. D, 37, 2078.

8. E. Turner, private communication (1989). 